\title{
Ação do fungo Metarhizium anisopliae sobre desenvolvimento do díptero Muscina stabulans em laboratório
}

\author{
[Action of Metarhizium anisopliae fungi over the development of Muscina stabulans dipteran in laboratory] \\ C.R. Zimmer ${ }^{1,2}$, M.C. Cárcamo $^{2}$, P.B. Ribeiro ${ }^{1,2}$, J.S. Nascimento ${ }^{3}$ \\ ${ }^{1}$ Faculdade de Agronomia "Eliseu Maciel" - UFPel \\ Caixa Postal 354 \\ 96010-900 - Pelotas, RS \\ ${ }^{2}$ Instituto de Biologia - UFPel - Pelotas, RS \\ ${ }^{3}$ Universidade Federal da Paraíba - João Pessoa, PB
}

\begin{abstract}
RESUMO
Avaliou-se o desenvolvimento de Muscina stabulans (Diptera, Muscidae) após exposição ao fungo Metarhizium anisopliae (isolado CG34), sob condições de laboratório. Suspensões de esporos foram preparadas nas concentrações de $10^{5}, 10^{6}, 10^{7}$ e $10^{8}$ conídeos $/ \mathrm{mL}^{-1}$. Noventa larvas pós-alimentar por tratamento, distribuídas em cinco tratamentos e três repetições, foram mergulhadas por um segundo nas respectivas suspensões, transferidas para placas de Petri com papel filtro umedecido e mantidas em estufa BOD à temperatura de $25^{\circ} \mathrm{C}$, umidade relativa de $80 \%$ e fotoperíodo de $12: 12$ horas até a emergência dos adultos. Foram avaliados a taxa de mortalidade pupal e o período de desenvolvimento pupal. As larvas apresentaram suscetibilidade à ação de $M$. anisopliae em diferentes concentrações. A mortalidade pupal assim como o tempo de desenvolvimento das pupas (de 8,8 a 10 dias) aumentaram conforme o aumento da concentração de conídios (de zero a 47\%). A aplicação de $M$. anisopliae nesta fase do ciclo biológico comprometeu o desenvolvimento de M. stabulans e interferiu no controle natural de pragas que se desenvolvem no mesmo ambiente.
\end{abstract}

Palavras-chave: mosca sinantrópica, controle biológico, controle microbiano

\begin{abstract}
The development of Muscina stabulans (Diptera, Muscidae) after exposure to Metarhizium anisopliae (CG34 isolated) fungi under laboratory conditions was evaluated. Suspensions of spores were prepared in concentrations of $10^{5}, 10^{6}, 10^{7}$, and $10^{8}$ conidia $/ \mathrm{mL}^{-1}$. Ninety postfeeding larvae per treatment, divided into five treatments and three repetitions, were dived for one second in the respective suspensions, and transferred to Petri dishes with humidified filter paper and maintained in BOD at $25^{\circ} \mathrm{C}$ with $80 \%$ relative humidity and a 12:12h photoperiod, until the emergency of adults. The rate of pupal mortality and the period of pupal development were evaluated. The larvae showed susceptibility to the action of $\mathrm{M}$. anisopliae in different levels of concentration. The pupal mortality increased accordingly to the increase in the conidia concentration (from zero to 47\%), as well as prolonged the time of development of the pupae (from 8.8 to 10 days). The application of $\mathrm{M}$. anisopliae in this phase of the biological cycle compromised the development of $\mathrm{M}$. stabulans and interfered in the natural control of pests that develop in the same environment.
\end{abstract}

Keywords: sinanthropic fly, biological control, microbial control

Recebido em 11 de fevereiro de 2009

Aceito em 30 de setembro de 2010

E-mail: crzimmerbio@yahoo.com.br 


\section{INTRODUÇÃO}

As moscas sinantrópicas são potenciais causadores de danos econômicos para criadores de animais, pois grande população se instala nesses locais, onde a oferta de substrato para o desenvolvimento larval é grande (Lomônaco e Prado, 1994), ocasionando incômodo e reduzindo a produtividade (Linhares, 1989). Programas de controle para moscas são normalmente baseados na utilização de produtos químicos. Porém, existem riscos potenciais tanto para o meio ambiente como para a saúde humana e ainda problemas relacionados ao desenvolvimento de resistência aos inseticidas (Howard e Wall, 1996). A necessidade de reduzir os impactos ambientais, causados pelo uso excessivo de agrotóxicos, tem motivado estudos de formas alternativas para controle de pragas, entre elas, a utilização de fungos entomopatogênicos.

A identificação de linhagens fúngicas patogênicas a moscas são de fundamental importância para estabelecer de medidas alternativas de controle de pragas sinantrópicas potenciais, assim como para conhecer os efeitos do fungo sobre insetos predadores que atuam como inimigos naturais dessas pragas. $\mathrm{O}$ fungo M. anisopliae é um componente natural da microbiota do solo pelo mundo inteiro e é agente da doença muscardine verde, que infecta insetos (Wright et al., 2004).

A suscetibilidade de muscídeos a fungos entomopatogênicos, particularmente Deuteromicetos (atuais mitospóricos), foi citada por Steinkraus et al. (1990) e, mais recentemente, por Renn et al. (1999), demonstrando o potencial patogênico como agentes de biocontrole para Musca domestica Linnaeus, 1758 (Diptera, Muscidae).

No Brasil, a utilização de fungos entomopatogênicos como agentes de controle biológico vem sendo aplicada utilizando-se principalmente as espécies $M$. anisopliae (Metchnikoff) (Mitospórico, Hyphomycetes) e Beauveria bassiana (Balsamo) (Ascomycata, Sordariomycetidae). Poucos são os estudos direcionados ao controle biológico de moscas por fungos entomopatogênicos (Senna-Nunes et al., 2002).
A "falsa mosca dos estábulos" como é popularmente conhecida, M. stabulans (Fallén, 1817) (Diptera, Muscidae), é um inseto de distribuição mundial (Skidmore, 1985), comumente encontrada em matéria orgânica animal em decomposição. As larvas são saprófagas, tornando-se carnívoras facultativas no terceiro ínstar, onde participam do controle natural de populações de algumas espécies de Diptera, que se desenvolvem no mesmo ambiente (Legner e Dietrich, 1989), como $M$. domestica (Skidmore, 1985).

O presente trabalho teve como objetivo avaliar o potencial patogênico e alguns efeitos subletais do isolado fúngico $M$. anisopliae (CG34) sobre larvas de terceiro estágio de $M$. stabulans em condições artificiais.

\section{MATERIAL E MÉTODOS}

Utilizou-se uma colônia de $M$. stabulans já adaptada às condições de laboratório, mantida durante todo o período de experimentação em câmara climatizada com temperatura de $26 \pm 2^{\circ} \mathrm{C}$, umidade relativa do ar acima de $75 \%$ e com fotofase de 12 horas, localizada no Laboratório de Biologia de Insetos no Instituto de Biologia (IB) da Universidade Federal de Pelotas. Os adultos foram mantidos em gaiolas teladas $(30 \times 30 \times 30 \mathrm{~cm})$ e alimentados com açúcar refinado e farinha de carne, na proporção de 2:1, respectivamente. A água foi oferecida em copos de becker com espuma de poliestireno cobrindo a superfície do líquido.

As larvas foram obtidas a partir de meio de cultura, exposto no interior das gaiolas, composto por farinha de carne e serragem na proporção de 2:1, adicionando-se água em quantidade suficiente para torná-lo pastoso. As posturas foram transferidas para um recipiente maior, contendo o mesmo meio, dentro de um funil de coleta. Nesse meio, após a eclosão, as larvas foram alimentadas até o terceiro ínstar, quando então abandonaram o substrato, e caíram em um recipiente contendo serragem úmida, para posterior pupariação.

O fungo M. anisopliae (CG34), preservado sob refrigeração em meio de cultivo batata-dextroseágar (BDA) e repicado para tubo de ensaio contendo o mesmo meio de cultivo, foi incubado em estufa a $25^{\circ} \mathrm{C}$, com fotoperíodo de $12 \mathrm{~h}$. Após 
a esporulação da cultura, foram feitas suspensões de esporos nas concentrações de $1,0.10^{5} ; 1,0.10^{6}$; $1,0.10^{7}$ e $1,0.10^{8}$ conídios. $\mathrm{mL}^{-1}$, em água destilada estéril adicionada de espalhante adesivo (Tween 80) na proporção de $0,01 \%$.

Após a obtenção, as larvas foram mergulhadas por um segundo nas respectivas suspensões, sendo o tratamento-controle composto apenas por água destilada estéril adicionada de $0,01 \%$ de Tween 80. Em seguida, foram acondicionadas em placas de Petri, forradas com papel absorvente previamente umedecido, em grupos de 30 larvas, sendo três repetições por tratamento, totalizando 90 larvas por tratamento, mantidas em estufa $\mathrm{BOD}$ à temperatura de $25^{\circ} \mathrm{C}$, umidade relativa de $80 \%$ e fotoperíodo de $12: 12 \mathrm{~h}$. O experimento foi avaliado diariamente até a emergência dos adultos para verificações dos possíveis efeitos subletais. As variáveis monitoradas foram mortalidade e período de desenvolvimento pupal.

Para o parâmetro período de desenvolvimento pupal, foi realizado o teste não paramétrico Kruskal-Wallis para verificação da disparidade entre os diferentes tratamentos, seguido de comparações entre as médias a $5 \%$ de significância.

\section{RESULTADOS E DISCUSSÃO}

A mortalidade de $M$. stabulans, cujas larvas foram tratadas com fungo entomopatogênico, demonstrou uma tendência crescente com o aumento das concentrações de $M$. anisopliae (CG34), em que a maior eficiência se deu na concentração de $1,0.10^{8}$ conídios. $\mathrm{mL}^{-1}$, tanto na mortalidade total como na mortalidade pupal (Tab. 1).

Tabela 1. Porcentagem de mortalidade e de indivíduos com defeitos em larvas, pupas e adultos anômalos de Muscina stabulans, cujas larvas foram tratadas com Metarhizium anisopliae em condições de laboratório

\begin{tabular}{cccccc}
\hline & \multicolumn{5}{c}{ Concentração de Metarhizium anisopliae } \\
\cline { 2 - 5 } & $\begin{array}{c}10^{0} * \\
(90)\end{array}$ & $\begin{array}{c}10^{5} \\
(90)\end{array}$ & $\begin{array}{c}10^{6} \\
(60)\end{array}$ & $\begin{array}{c}10^{7} \\
(90)\end{array}$ & $\begin{array}{c}10^{8} \\
(90)\end{array}$ \\
\hline $\begin{array}{c}\text { Mortalidade total } \\
(\%)\end{array}$ & 0 & 3,30 & 25,00 & 20,00 & 50,00 \\
$\begin{array}{c}\text { Mortalidade pupal } \\
(\%)\end{array}$ & 0 & 3,30 & 6,20 & 5,30 & 47,10 \\
$\begin{array}{c}\text { Indivíduos com } \\
\text { defeitos }(\%)\end{array}$ & - & - & 4,45 & 5,56 & - \\
\hline
\end{tabular}

*Água destilada estéril adicionada de $0,01 \%$ de Twenn 80 .

Valor entre parênteses refere-se ao número de larvas utilizadas no tratamento.

Mortalidade total: mortalidade de larvas mais pupas.

A suscetibilidade de distintas espécies de moscas a diferentes fungos entomopatogênicos foi demonstrada por diversos autores, como o potencial patogênico do fungo $M$. anisopliae sobre M. domestica (Barson et al., 1994; Bernardi et al., 2006), em adultos de $M$. domestica e Bactrocera tryoni (Frogatt) (Diptera, Tephritidae) (Carswel et al., 1998), em ovos, pupas e adultos da mosca do chifre, Haematobia irritans (L.) (Diptera, Muscidae) (AngelSahagun et al., 2005), somente adultos de $H$. irritans (Lohmeyer e Miller, 2006) e também em espécies da família Calliphoridae como Lucilia sericata (Wright et al., 2004). O fungo $B$. bassiana também apresenta patogenicidade sobre dípteros como M. domestica (Watson et al., 1995; Angel-Sahagun et al., 2005), e ainda existem citações para os fungos Aspergillus flavus e Penicillium corylophilum sobre adultos de M. domestica (Senna-Nunes et al., 2002) e Paecilomyces fumosoroseus patogênico para $H$. irritans (Angel-Sahagun et al., 2005).

Em pesquisa com fungos entomopatogênicos em solos britânicos foi apontado que as espécies mais comuns foram $B$. bassiana e Paecilomyces farinosus, mas com maior prevalência em amostras de solo de florestas e não de solos com características de cultivo (Chandler et al., 1997). Porém, depois de uma inoculação inicial, $B$. bassiana apresentou persistência baixa no solo (Vänninen et al., 2000). Em comparação, $M$. anisopliae persistiu extremamente bem e, depois de três anos, permaneceu em números suficientes 
de propágulos infecciosos, infectando mais de $80 \%$ de larvas de Tenebrio molitor (Linnaeus) (Coleoptera, Tenebrionidae) usadas como iscas no solo. A concentração mais alta de $M$. anisopliae foi encontrada nos $5 \mathrm{~cm}$ superiores da camada do solo (Vänninen et al., 2000). Esses mesmos autores salientaram, que durante o inverno, os fungos entomopatogênicos permanecem viáveis, até mesmo quando a temperatura do solo cai a $0^{\circ} \mathrm{C}$ e a temperatura do ar a $-25^{\circ} \mathrm{C}$, mas sem evidências de que eles, neste solo, são capazes de infectar os insetos nesta fase.

O isolado fúngico CG34 de $M$. anisopliae promoveu mortalidade total de pupas de terceiro ínstar pós-alimentar que variou de 3,3\% a 50,0\%, respectivamente, para as concentrações de $1,0.10^{5}$ e $1,0.10^{8}$ conídios. $\mathrm{mL}^{-1}$, sugerindo uma relação diretamente proporcional (Tab. 1). Os valores obtidos de mortalidade são, em geral, comparáveis aos observados por outros autores, em que o grau de toxicidade depende da fase de desenvolvimento do inseto, do ínstar, da concentração da suspensão fúngica utilizada no bioensaio e do tipo e tempo de exposição em que o inseto foi submetido ao tratamento.

Barson et al. (1994), ao avaliarem o potencial entomopatogênico de seis espécies de fungos, citaram M. anisopliae como a espécie com maior potencial patogênico sobre larvas de terceiro ínstar de $M$. domestica, quando estas foram mergulhadas em suspensões de $1,0.10^{6}$ e $1,0.10^{5}$ conídios. $\mathrm{mL}^{-1}$, emergindo apenas 1,0 e $16,0 \%$, respectivamente, sendo que, com o aumento da concentração, ocorreu a morte de todas as larvas.

Wright et al. (2004), ao testarem M. anisopliae como agente potencial de biocontrole contra fêmeas adultas Lucilia sericata (Meigen) (Diptera, Calliphoridae), obtiveram, em teste com suspensão de esporos em óleo de silicone, porcentagens mais altas de infecção (50-70\%) do que os formulados em $0,3 \mathrm{~g} \mathrm{~L}^{-1}$ do detergente Tween 80 (10-20\%). A concentração de esporo mostrou efeito significativo sobre a infecção de moscas, com índices mais altos de mortalidade $(64 \%)$ na suspensão de $1,0.10^{7}$ conídios. $\mathrm{mL}^{-1}$.

Bernardi et al. (2006) verificaram que o isolado de M. anisopliae (CG34) apresentou potencial entomopatogênico sobre as pupas de $M$. domestica, isto é, $68 \%$ de mortalidade na concentração de $1,0.10^{7}$ conídios. $\mathrm{mL}^{-1}$. Já o isolado de B. bassiana (CG240) resultou em somente $2 \%$ de mortalidade na mesma concentração.

As concentrações $1,0.10^{6}$ e $1,0.10^{7}$ conídios. $\mathrm{mL}^{-1}$ apresentaram considerável porcentagem de indivíduos com má-formação de asas, 4,4\% e $5,6 \%$, respectivamente (Tab. 1). A taxa de indivíduos malformados somados à taxa de mortalidade total mostram, que nas concentrações $1,0.10^{6}$ e $1,0.10^{7}$ conídios. $\mathrm{mL}^{-1}$, o índice de mortalidade seria elevado para $29,4 \%$ e 25,6\%, respectivamente, uma vez que indivíduos com anomalias de asas são incapazes de voar, consequentemente não se reproduzem e, assim, tornam-se moscas inviáveis.

O período de desenvolvimento pupal diferiu significativamente entre os tratamentos $(\mathrm{P}<0,001)$ (Fig. 1), isto é, os tratados foram mais tardios que o do grupo-controle. No entanto, nas três concentrações intermediárias $\left(1,0.10^{5}\right.$; $1,0.10^{6}$ e $1,0.10^{7}$ conídios. $\mathrm{mL}^{-1}$ ), não houve diferença significativa entre si, enquanto a maior concentração $\quad\left(1,0.10^{8} \quad\right.$ conídios. $\left.\mathrm{mL}^{-1}\right)$ foi diferente de todos os outros tratamentos. O tempo de desenvolvimento foi diretamente proporcional ao aumento da concentração fúngica de $M$. anisopliae, isto é, a diferença entre o controle e o tratamento com maior período de desenvolvimento pupal foi de, aproximadamente, um dia.

O aumento no período de desenvolvimento, diretamente relacionado com o aumento da concentração de $M$. anisopliae e, consequentemente, com a mortalidade, pode ser atribuído à ação do fungo, que aumenta com o passar do tempo (Senna-Nunes et al., 2002), e à velocidade do processo germinativo, que depende do isolado fúngico, porém, em geral, estão envolvidos processos importantes para que ocorra a penetração e a colonização do fungo. Uma certa densidade de conídios é requerida para que ocorra a penetração efetiva do fungo na cutícula (Zhioua et al., 1997), como um mecanismo de ação em massa, o que explica a baixa patogenicidade encontrada nas suspensões de menor concentração de conídios. 


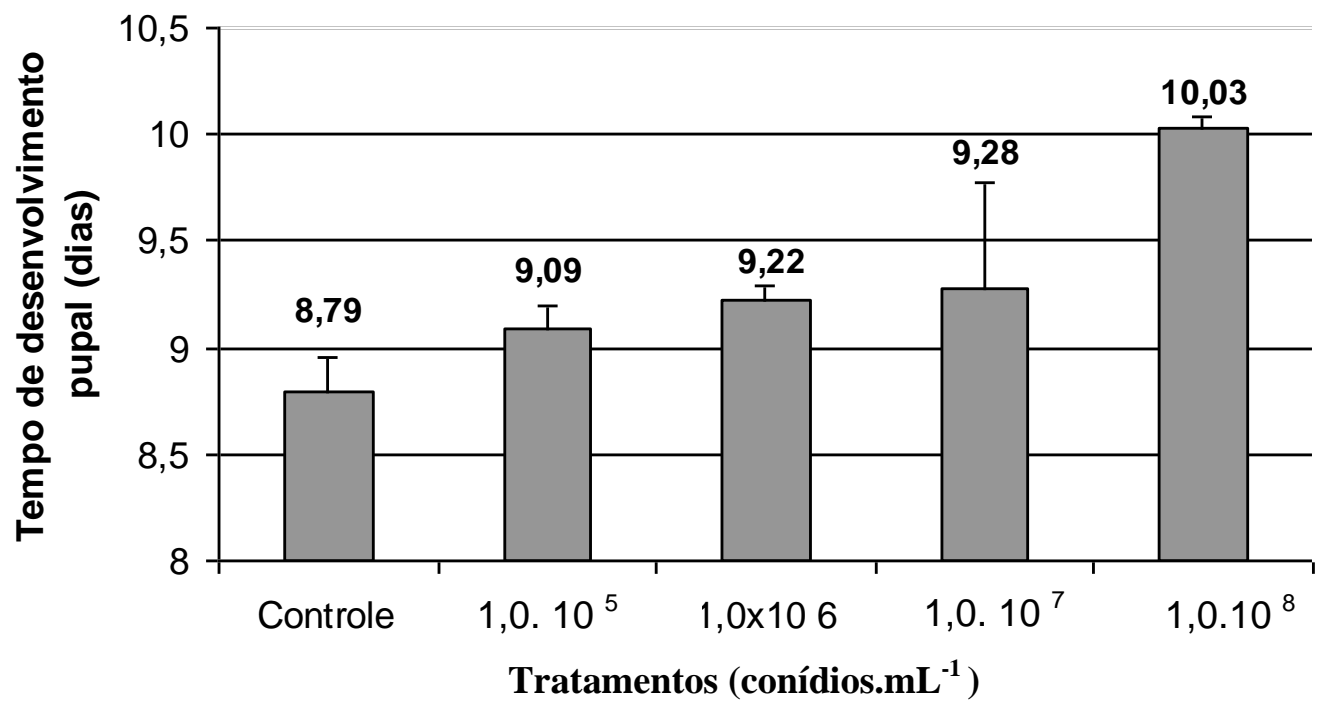

Figura 1. Tempo de desenvolvimento de pupas de Muscina stabulans, de acordo com a concentração de Metarhizium anisopliae em condições de laboratório. As barras representam o desvio-padrão da média.

Em situações adversas, os dípteros tendem a prolongar o tempo de desenvolvimento para completar seu ciclo (Mueller et al., 2005; Zimmer et al., 2006), e, com o aumento do período pupal, esses indivíduos permaneceriam mais tempo no ambiente de desenvolvimento, na fase de pupa, que, por se tratar de estádio imóvel, torna o inseto mais sensível a condições desfavoráveis.

Se se considerar o efeito cumulativo de mortalidade observado no experimento, juntamente com o prolongamento do período de desenvolvimento, constata-se que a patogenicidade de $M$. anisopliae foi efetiva contra larvas de terceiro estágio de $M$. stabulans. Desse modo, são necessários estudos adicionais com diferentes concentrações fúngicas, diferentes formas de aplicação, testes com outros estádios de desenvolvimento para, assim, entender o impacto do fungo entomopatogênico e sua dinâmica sobre a população de insetos não alvo, que apresentam relevante importância no controle natural de insetos praga como $M$. domestica.

\section{CONCLUSÕES}

Pode-se concluir que o fungo $M$. anisopliae isolado CG34 exerce atividade patogênica sobre larvas L3 de M. stabulans, reduz a viabilidade de pupas e aumenta o período de desenvolvimento pupal, indicando que a aplicação do fungo, nesta fase do ciclo biológico da mosca, compromete o seu desenvolvimento e interfere no controle natural que o inseto realiza sobre espécies pragas que se desenvolvem no mesmo ambiente.

\section{AGRADECIMENTOS}

À CAPES, pela bolsa de doutorado ao primeiro autor e bolsa de mestrado concedida ao segundo autor. Aos biólogos Juliano Lessa Pinto Duarte, pelas correções no abstract e Eduardo Bernardi, pelo auxílio na preparação das suspensões fúngicas.

\section{REFERÊNCIAS BIBLIOGRÁFICAS}
ANGEL-SAHAGÚN, C.A.; LEZAMA- GUTIÉRREZ, R.; MOLINA-OCHOA J. et al. Susceptibility of biological stages of the horn fly, Haematobia irritans, to entomopathogenic fungi (Hyphomycetes). J. Insect Sci., v.5, p.1-8, 2005.

BARSON, G.; RENN, N.; BYWATER, A. Laboratory evaluation of six species of entomopathogenic fungi for the control of the house fly (Musca domestica L.), a pest of intensive animal units. J. Inverteb. Pathol., v.64, p.107-113, 1994. 
BERNARDI, E.; PINTO, D.M.; NASCIMENTO, J.S. et al. Efeito dos fungos entomopatogênicos Metarhizium anisopliae e Beauveria bassiana sobre o desenvolvimento de Musca domestica L. (Diptera: Muscidae) em laboratório. Arq. Inst. Biol., v.73, p.127-129, 2006.

CARSWELL, R.; SPOONER-HART; MILNER, R.J. Laboratory susceptibility of Musca domestica L. (Diptera: Muscidae) and Bactrocera tryoni (Frogatt) (Diptera: Tephritidae) to an isolate of Metarhizium anisopliae (Metsch.) Sorokin Isla. Aust. J. Entomol., v.37, p.1-284, 1998.

CHANDLER, D.; HAY, D.; REID, A.P. Sampling and occurrence of entomopathogenic fungi and nematodes in UK soils. Appl. Soil Ecol., v.5, p.133-141, 1997.

HOWARD, J.; WALL, R. Control of the house fly, Musca domestica, in poultry units: current techniques and future prospects. Agr. Zool. Rev., v.7, p.247-265, 1996.

LEGNER, E.F.; DIETRICH, E.J. Coexistence of predatory Muscina stabulans and Ophyra aenescens (Diptera: Muscidae) with dipterous prey in poultry manure. Entomophaga, v.34, p.453-461, 1989.

LINHARES, A.X. Perspectivas em controle biológico de dípteros muscoides. In: SEMINÁRIO SOBRE INSETOS E ÁCAROS, 3., 1989, Campinas, SP. Anais... Campinas, SP: Sociedade Entomológica do Brasil, 1989. p.123133. (Resumo).

LOHMEYER, K.H.; MILLER, J.A. Pathogenicity of three formulations of entomopathogenic fungi for control of adult Haematobia irritans (Diptera: Muscidae). J. Econ. Entomol., v.99, p.1943-1947, 2006.

LOMÔNACO, C.; PRADO, A.P., Estrutura comunitária e dinâmica populacional da fauna de dípteros e seus inimigos naturais em granjas avícolas. An. Soc. Entomol. Brasil, v.23, p.71-80, 1994.

MUELLER, L.D.; FOLK, D.G.; NGUYEN, N. et al. Evolution of larval foraging behaviour in Drosophila and its effects on growth and metabolic rates. Physiol. Entomol., v.30, p.262269, 2005.
RENN, N.; BYWATER A.F.; BARSON, G. A bait formulated with Metarhizium anisopliae for the control of Musca domestica L. (Diptera: Muscidae) assessed in large-scale laboratory enclosures. J. Appl. Entomol., v.123, p.309-314, 1999.

SENNA-NUNES, M.; COSTA, G.L.; BITTENCOURT, V.R.E.P. et al. Avaliação in vitro dos fungos Aspergillus flavus e Penicillium corylophilum em larvas de Musca domestica (Diptera: Muscidae). Parasitol. Latinoam., v.57, p.9-14, 2002.

SKIDMORE, P. The biology of the Muscidae of the world. Dordrecht: Dordrecht Kunk, 1985. $550 \mathrm{p}$.

STEINKRAUS, D.C.; GEDEN, C.J.; RUTZ, D.A. et al. First report of the natural occurrence of Beauveria bassiana (Moniliales Maniliaceae) in Musca domestica (Diptera, Muscidae). J. Med. Entomol., v.27, p.309- 312, 1990.

WRIGHT, C.; BROOKS, A.; WALL, P.R. Toxicity of the entomopathogenic fungus, Metarhizium anisopliae (Deuteromycotina: Hyphomycetes) to adult females of the blowfly Lucilia sericata (Diptera: Calliphoridae). Pest. Manag. Sci., v.60, p.639-644, 2004.

VÄNNINEN, I.; TYNI-JUSLIN, J.; HOKKANEN, H. Persistence of augmented Metarhizium anisopliae and Beauveria bassiana in Finnish agricultural soils. Biocontrol, v.45, p.201-222, 2000.

WATSON, D.W.; GEDEN, C.J.; LONG, S.J. et al. Efficacy of Beauveria bassiana for controlling the house fly and stable fly (Diptera: Muscidae). Biol. Control, v.5, p.405-411, 1995.

ZHIOUA, E.; BROWNING, M.; JOHNSON, P.W. et al. Pathogenicity of the entomopathogenic fungs Metarhizium anisopliae (Deuteromycetes) to Ixodes acapularis (Acari: Ixodidae). J. Parasitol., v.83, p.815-818, 1997.

ZIMMER, C.R.; PIRES, S.M.; CÁRCAMO, M.C. et al. Efeitos da competição larval intraespecífica sobre caracteres biométricos de Muscina stabulans (Fallén, 1817) (Diptera: Muscidae) em laboratório. Arq. Inst. Biol., v.73, p.203-209, 2006. 